\title{
EFECTO DE UN PROMOTOR NATURAL DE CRECIMIENTO SOBRE LA RESPUESTA PRODUCTIVA DE Cavia porcellus "CUYES" EN LA ETAPA DE CRECIMIENTO - ACABADO
}

\section{EFFECT OF A NATURAL GROWTH PROMOTER ON THE PRODUCTIVE RESPONSE OF Cavia porcellus "CUYES" IN THE GROWTH STAGE - FINISHING}

\author{
(iD) Víctor Pretel-Jesús ${ }^{1}$ (iD Julia Mercedes Ramírez-Sánchez ${ }^{1}$ \\ (iD) Alejandra Silva- Santisteban ${ }^{1}$ \\ ${ }^{1}$ Universidad Nacional de Trujillo, Trujillo, Perú. \\ Correspondencia: \\ Dra. Julia M. Ramírez Sánchez \\ jramirez@unitru.edu.pe
}

\section{RESUMEN}

El estudio tuvo como objetivo evaluar el efecto de la adición de un promotor natural de crecimiento (PNC) sobre la respuesta productiva de cuyes mejorados en la etapa de crecimiento - acabado. Se utilizaron 72 cuyes destetados ( $21 \pm 2$ días) de ambos sexos distribuidos en un diseño de bloques completamente al azar con tres tratamientos de 24 cuyes por tratamiento bloqueados por sexo. Los tratamientos fueron: TO: ración con inclusión de APC (50 g de zinc bacitracina /100 kg); T1: ración con inclusión de PNC (50 g/100 kg) y T2: ración con inclusión de PNC (30 g/100 kg). Se evaluaron los parámetros: incrementos de peso (IDP), conversión alimenticia (CA), rendimiento de carcasa (RDC) y porcentaje de mortalidad. Los datos se analizaron con la prueba de homogeneidad de varianzas de Lévenne, ANOVA y prueba de Duncan. Los resultados de IDP el T1 presentó 841,5 g y el T2 con 799,6 g; siendo estadísticamente iguales pero superiores al T0 con 698,6 g presentando diferencias estadísticas altamente significativas ( $p<0,01$ ); para CA los resultados fueron de 3,1 3,2 y 3,3 para T1, T2 y T0 respectivamente sin diferencias estadísticas ( $p>0,05)$; el mayor RDC se logró en T1 (71.5 \%) luego el T2 (70.2 \%) y el T0 (70 \%) sin diferencias estadísticas y T2 presentó el mayor porcentaje de mortalidad (16.7\%) seguido del T0 (8.3 \%) y el T1 $(0 \%)$ igualmente sin diferencias. Se concluye según los resultados obtenidos que la inclusión de $50 \mathrm{~g} / 100 \mathrm{~kg}$ de PNC (sin APC) tuvo el mejor efecto sobre el rendimiento productivo de cuyes en la etapa crecimiento - engorde.

Palabras clave: Cuyes, parámetros productivos, promotor natural de crecimiento.

\section{ABSTRACT}

The objective of the study was to evaluate the effect of the addition of a natural growth promoter (PNC) on the productive response of improved guinea pigs in the growth-finishing stage. 72 weaned guinea pigs ( $21 \pm 2$ days) of both sexes were 
used, distributed in a completely randomized block design with three treatments of 24 guinea pigs per treatment blocked by sex. The treatments were: TO: ration with inclusion of APC (50 g of zinc bacitracin / $100 \mathrm{~kg}$ ); T1: ration including PNC $(50 \mathrm{~g} /$ $100 \mathrm{~kg})$ and T2: ration including PNC $(30 \mathrm{~g} / 100 \mathrm{~kg})$. The parameters were evaluated: weight increases (IDP), feed conversion (CA), carcass yield (RDC) and percentage of mortality. Data were analyzed with Lévenne's test of homogeneity of variances, ANOVA and Duncan's test. The results of IDP the T1 presented $841.5 \mathrm{~g}$ and the T2 with $799.6 \mathrm{~g}$; being statistically equal to but higher than T0 with $698.6 \mathrm{~g}$ presenting highly significant statistical differences $(p<0.01)$; for CA the results were 3.1, 3.2 and 3.3 for T1, T2 and T0 respectively without statistical differences $(p>0.05)$; the highest RDC was achieved in T1 (71.5 \%) then T2 (70.2\%) and T0 (70 \%) without statistical differences and T2 presented the highest percentage of mortality (16.7 $\%)$ followed by TO (8.3\%) and T1 (0 \%) equally without differences. According to the results obtained, it is concluded that the inclusion of $50 \mathrm{~g} / 100 \mathrm{~kg}$ of PNC (without APC) had the best effect on the productive performance of guinea pigs in the growthfattening stage.

Keywords: Guinea pigs, productive parameters, natural growth promoter.

\section{INTRODUCCIÓN}

El cuy (Cavia porcellus), representa una significativa fuente de proteína para el habitante andino. Su fácil crianza y su alto requerimiento hace que esté en continuo incremento frente a otras especies animales de interés pecuario (Pozo y Tepú, 2012)

El cuy es una especie animal de alto valor nutritivo que apoya con la seguridad alimentaria de la población rural de escasos recursos económicos. En los países andinos como el nuestro se encuentra una población estable de más o menos 35 millones de cuyes. En el Perú, tenemos la mayor población y consumo de cuyes, se registra una producción anual de 16,500 toneladas de carne proveniente del beneficio de más de 65 millones de cuyes, producidos por una población más o menos estable de 22 millones de animales criados mayormente con sistemas de producción familiar (Otero, 2013).

La Organización Mundial de la Salud define el término agente promotor del crecimiento como sustancias diferentes de los nutrientes de la dieta que incrementan el ritmo de crecimiento y mejoran el índice de conversión de los animales en buen estado de salud y con sus requerimientos nutritivos cubiertos. Por esta razón, el término promotor del crecimiento se puede aplicar a más de un tipo de sustancias utilizadas en producción animal. La ganancia de peso en cuyes es un factor principal y determinante para la posterior comercialización de los mismos, la no adición de promotores de crecimiento en la dieta del cuy prolonga el periodo de crecimiento provocando un incremento de los costos de producción. Los aditivos alimenticios son sustancias que se agregan a los alimentos intencionadamente para modificar sus propiedades, técnicas de elaboración, conservación o mejorar su adaptación al uso a que estén destinados. En ningún caso tienen un papel enriquecedor del alimento (Coba, 2012).

En la actualidad la producción animal, se enfrenta a un futuro sin antibióticos, el aditivo más eficiente que se tenía. La prohibición de la Unión Europea, y el retiro gradual de los antibióticos en la alimentación animal en otras partes del mundo, ha puesto una presión adicional a favor de la mejora de salud intestinal y el bienestar de los animales. Actualmente hay un aumento en las investigaciones para evaluar productos alternativos para mantener la flora intestinal y la salud digestiva, donde incluye diversas clases de productos como prebióticos; probióticos, ácidos orgánicos, enzimas. Para poder ser aceptados por la industria, los efectos de estos productos deben ser demostrados por una mejora de los rendimientos productivos alcanzados de los animales similar los que se 
alcanza con los antibióticos (Ravidran, 2010).

Al igual que otras especies, los nutrientes que necesita el cuy son: agua, proteína, fibra, energía, ácidos grasos esenciales, minerales y vitaminas. Los requerimientos dependen de la edad, estado fisiológico genotipo y medio ambiente donde se desarrolle la crianza. Los requerimientos nutricionales necesarios para lograr mayores crecimientos han sido desarrollados con la finalidad de encontrar porcentajes adecuados de proteína, así como los niveles de energía. Por su sistema digestivo el régimen alimenticio que reciben los cuyes es a base de forraje más un suplemento (Castro, 2002).

La crianza de cuyes está entrando a una nueva etapa que es la de constituirse en una alternativa alimenticia no solo en nuestro país sino fuera de él, bajo esta perspectiva, su explotación representa una oportunidad de inversión. En zonas rurales el cuy es considerado fuente de ingresos al servir como medio de intercambio por otras mercancías (trueque) o por venta directa para complementar la dieta familiar y cubrir algunos gastos de educación de los hijos (Rey, 2008).

Una de las formas más comunes para el control de la salmonelosis es la utilización de antibióticos como preventivo en las unidades de producción de cuyes. Pero, el uso no controlado de los antibióticos tiene efectos negativos para la salud del ser humano por la trasmisión de sus residuos que puede presentar la carne del cuy. Existe, además, un riesgo muy alto de generar resistencia de la salmonella a los antibióticos en perjuicio de la salud animal (Phillips et al., 2004; Cano, 2012). Los problemas asociados al uso de antibióticos para garantizar la producción de animales, permitió la búsqueda de nuevas alternativas más promisorias como el uso de probióticos (Morales, 2017).

El promotor natural de crecimiento (PNC), utilizado en el presente estudio (Turbo Plus 80) presenta la siguiente composición: Lactosa, sulfato de sodio anhidro, cloruro de potasio, cloruro de sodio. Probiótico concentrado que contiene los microorganismos: Streptococus faccium, Streptoccus thermophilum,
Lactobacillus bulgaricus, Lactobacillis acidoplillus, Lactobacillus casei, Bifidobacterium bifidum, Lactobacillus plantarum, Aspergillus oryzae y Torulopsis bovina; Vitamina A, Niacinamida, L-Lisina, Sulfato de manganeso. Monohidratado, Vitamina B12 Cristalina, DLMetionina, Pantotenato Calcio. Dióxido de silicio, Riboflavina, Sorbato de Potasio (inhibidor de hongos), Colecalciferol, Piridoxina HCL, Ácido Ascórbico, Ácido Fólico; gracias a este balance de componentes lo hace un producto ideal para las etapas de stress intensos producidos por los altos y bajos cambios climáticos. Es un reconstituyente, el cual también contiene el $80 \%$ de aminoácidos lo que ayuda a mejorar en menor tiempo la condición del animal, porque no es solo agregarle vitaminas, si no nutrirlo y así elevar la inmunidad y como consecuencia mejorar sus parámetros productivos. Esta investigación se basa en la bibliografía encontrada y con la finalidad de evaluar la respuesta productiva de Cavia porcellus (cuyes) a la adición de un promotor natural de crecimiento mejorados en la etapa de crecimiento - acabado.

\section{MATERIALES Y MÉTODOS}

La investigación fue realizada en instalaciones de una crianza familiar, en el distrito de Laredo provincia de Trujillo; Departamento de La Libertad. Ubicado a una latitud de -8.08965 y una longitud: -78.9602, cuya temperatura generalmente varía de $17{ }^{\circ} \mathrm{C}$ a $26{ }^{\circ} \mathrm{C}$ y $74 \%$ de humedad relativa.

Para la realización del experimento se utilizaron 72 cuyes destetados ( $21 \pm 2$ días) de genética mejorada cuya alimentación fue a base de forraje verde (maíz chala) y concentrado. En la metodología experimental fueron considerados 3 tratamientos, con 24 cuyes en cada uno, cada tratamiento con 2 bloques (sexo) y 3 repeticiones con 4 cuyes por repetición haciendo un total de 24 cuyes por tratamiento. Siendo la distribución de los tratamientos como se describen a continuación: T0: Testigo; Forraje verde + concentrado con APC $(50 \mathrm{~g} / 100 \mathrm{~kg}$ de concentrado de zinc bacitracina); T1: Forraje verde + concentrado con $50 \mathrm{~g} / 100 \mathrm{~kg}$ de alimento de Promotor Natural de Crecimiento (PNC) sin APC y T2: Forraje verde + concentrado con $30 \mathrm{~g} / 100 \mathrm{~kg}$ alimento de Promotor Natural 
de Crecimiento (PNC) sin APC.

En cuanto a las instalaciones las paredes fueron de ladrillo con techo parcialmente cubierto con planchas de fibrocemento lo que aseguraba luminosidad y ventilación adecuada. Las pozas para alojar a los cuyes fueron de adobe con dimensiones de $1 \mathrm{~m}$ de largo por $0,75 \mathrm{~m}$ de ancho y una altura de $0,40 \mathrm{~m}$ las mismas que alojaron a cada grupo de cuatro cuyes de cada una de las repeticiones pertenecientes a cada tratamiento los cuales fueron asignados al azar. Antes del inicio del experimento, todas las pozas fueron limpiadas y desinfectadas para evitar problemas sanitarios; cada poza contaba con un bebedero de arcilla de 0,5 I de capacidad y un comedero del mismo material de $0.5 \mathrm{~kg}$ de capacidad. La identificación de cada animal, se realizó en base a las características de color de cada individuo. La fase experimental tuvo un periodo de duración de 90 días.

La alimentación estuvo conformada por forraje verde (maíz chala) y concentrado con 18,09\% de proteína y 2,850 Mcal/kg de alimento de ED y APC o PNC según fuera el caso. El suministro de alimento (FV + concentrado) se realizó diariamente dos veces al día (mañana y tarde) previo lavado de los comederos, desde el inicio hasta el final del experimento; tomándose registro diario de la cantidad otorgada y del residuo. Los bebederos previa limpieza, fueron abastecidos diariamente con agua limpia y fresca para su consumo ad libitum.

Para la toma de datos y su posterior evaluación se registró semanalmente el peso individual por muestreo al azar, así como el consumo semanal y el número de cuyes muertos o enfermos por tratamiento.

Las variables evaluadas fueron el peso inicial (PI), incremento de peso (IDP), conversión alimenticia (CA), rendimiento de carcasa (RDC) y porcentaje de mortalidad.

Para el procesamiento y análisis de los datos se utilizó el programa estadístico SPSS versión 24 para Windows. Se utilizó el análisis de varianza del Diseño de Bloques Completamente al Azar y de encontrarse diferencias significativas entre las medias de los tratamientos se aplicó la prueba de Duncan al 0,05 nivel de significancia.

\section{RESULTADOS Y DISCUSION Pesos iniciales e incrementos de peso}

Los pesos medios iniciales para TO fue 317,3 g; para T1 fue 302,3 g y para T2 fue 276,3 g; para los que se realizó la prueba de homogeneidad de varianzas (prueba de Levene), determinándose que los pesos de los tratamientos fueron homogéneos $(p>0,05)$. Lo que nos dice que, a pesar de la no uniformidad de tamaño de camada, línea genética, ambiente de manejo y alimentación los pesos fueron homogéneos, por lo que podemos deducir que influyó en estos pesos la calidad de la leche materna y la suplementación, conforme con lo reportado por Aliaga et al. (2009) y Morales (2009).

En la presente investigación T1 logró un mayor incremento de peso con una media de $841,5 \mathrm{~g}$ seguido del T2 con una media de 799,6 g y el T0 con $698,6 \mathrm{~g}$. Por otra parte, los cuyes machos tuvieron un mayor incremento de peso con una media de $814,3 \mathrm{~g}$ y las hembras con $750 \mathrm{~g}$. Se encontraron diferencias estadísticas altamente significativas $(p<0,01)$ entre los tratamientos, pero no entre el sexo de los cuyes $(p>0,05)$. Resultados similares fueron reportados por Torres et al. (2013), quienes en un experimento con cuyes utilizaron 5 tratamientos, T1, T2 y T3 recibieron 100, 150 y $200 \mathrm{ml}$ de probiótico, respectivamente, y T4 y T5 fueron los controles positivos (dieta base + antibiótico) y negativo (dieta base), respectivamente. El consorcio probiótico fue elaborado con seis especies bacterianas: Enterococos hirae, Lactobacillus reuteri, Lactobacillus frumenti, Lactobacillus johnsoni, Streptococcus thoraltensis, Bacillus pumilus. El tratamiento $\mathrm{T} 1$ tuvo $717,19 \mathrm{~g}$ de ganancia, T2 con 654,78 g, T3 fue de 695,13 g, T4 con 616,09 g y el T5 con 659,89 g. aunque no se reportaron diferencias estadísticas significativas. Resultados distintos fueron hallados por Guevara y Carcelén (2014), ellos reportaron que la mayor ganancia de peso lo presentaron los cuyes del T3. Dieta + levadura con 493,67g y la menor ganancia lo presentaron los cuyes del T4. Dieta + Lactobacillus + levadura con 474,25 g, aunque estos no alcanzaron a ser estadísticamente diferentes. 


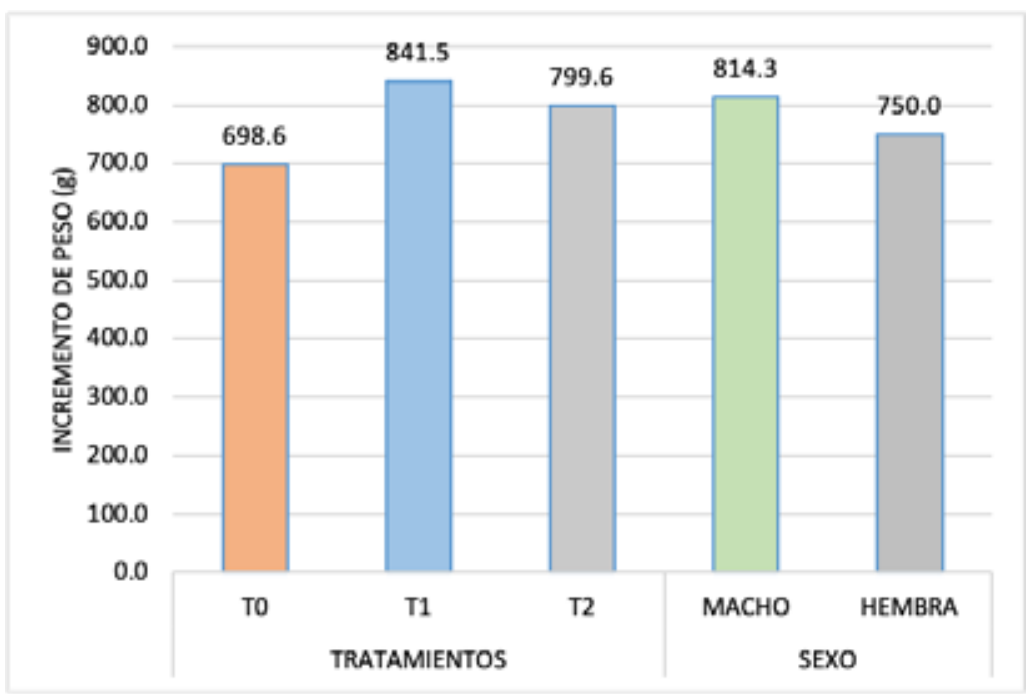

Figura 1. Incrementos de peso por tratamientos y por sexo.

\section{Conversión alimenticia}

En esta investigación, el T1 tuvo la mejor conversión alimenticia con una media de 3,1 seguido del T2 con una media de 3,2 y finalmente el T0 con una media de 3,3. Por otro lado los cuyes machos tuvieron la mejor conversión alimenticia con una media de 3,2 y las hembras con una media de 3,3; no se encontraron diferencias estadísticas $(p>0,05)$ entre los tratamientos ni entre el sexo de los cuyes. Resultados parecidos fueron reportados por Guevara y Carcelén (2014), quienes mencionaron que la mejor conversión alimenticia lo lograron los cuyes del T3 Dieta + levadura con 4,4 y la menor los cuyes del T2 Dieta + Lactobacillus y T4. Dieta + Lactobacillus + levadura con 4,6. No existe diferencia estadística significativa. Torres et al. (2013), tuvieron conversiones alimenticias de 4,33 en T1, 3,90 en T2, 4,33 para el T3, 4,43 en el T4 y 5,04 en T5. Encontraron diferencias estadísticas significativas. Resultados distintos fueron obtenidos por Cano et al. (2015), quienes mostraron que los niveles del probiótico redujeron significativamente $(P=0.0002)$ el índice de conversión alimenticia.

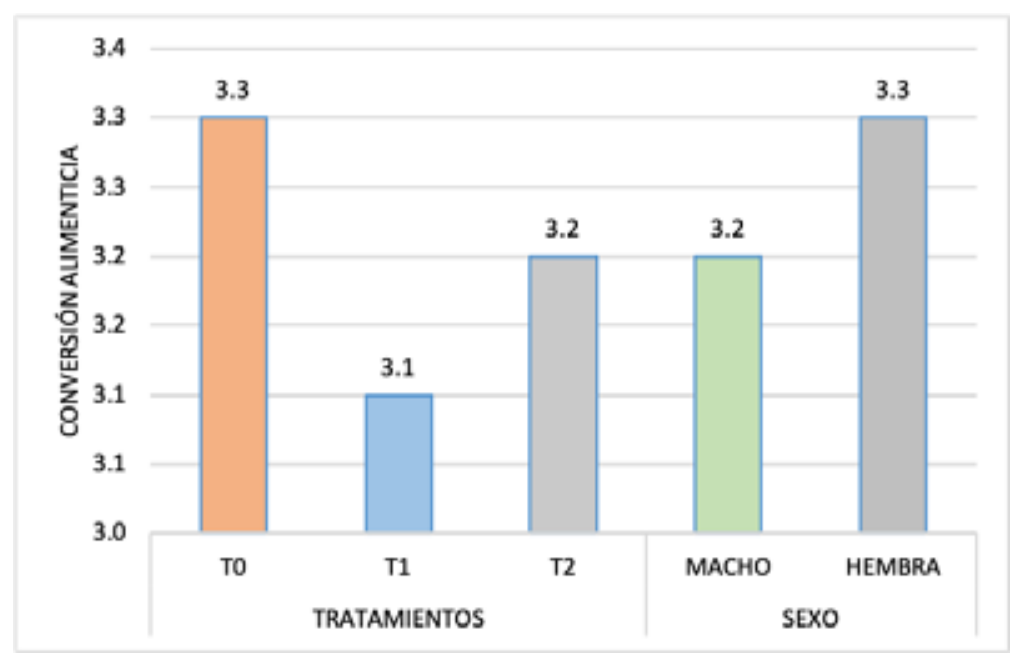

Figura 2. Conversión alimenticia por tratamientos y por sexo 


\section{Rendimiento de carcasa}

Se calculó que el mayor rendimiento de carcasa lo logró el T1 (71,5 \%) seguido por el T2 (70,2 $\%)$ y finalmente el TO (70 \%). Los cuyes machos lograron el mayor rendimiento de carcasa en el TO $(72,1 \%)$, el mayor valor registrado en hembras fue en el T1 (71,4\%). No se hallaron diferencias estadísticas $(p>0,05)$ entre los tratamientos ni entre el sexo de los cuyes. Resultados parecidos fueron obtenidos por Cano et al. (2015), quienes tuvieron en el tratamiento suplementado con $100 \mathrm{ml}$ de probiótico un rendimiento de carcasa de $70,88 \%$, el tratamiento suplementado con $150 \mathrm{ml}$ de probiótico tuvo un valor de 72.02 $\%$, el tratamiento suplementado con $200 \mathrm{ml}$ de probiótico tuvo un valor de $71,17 \%$ y el tratamiento control tuvo un rendimiento de carcasa de 66,62 \%. No encontraron diferencias estadísticas significativas. Estos resultados concuerdan con los hallados por Torres et al. (2013), quienes tampoco encontraron diferencias estadísticas respecto al peso de la carcasa entre grupos experimentales cuando evaluó el uso de diferentes niveles de probióticos en la alimentación de cuyes, siendo el promedio general de $707,4 \mathrm{~g}$ y el rendimiento porcentual de la carcasa fue de 71,7 $\pm 2,6 \%$. Estos resultados son similares a los obtenidos por Andía y Ángeles (2021), cuyos resultados mostraron que la inclusión de una mezcla probiótica de Lactobacillus acidophilus, L. casei y Saccharomyces cerevisiae en la alimentación del cuy, tuvo efecto en el consumo de alimento y rendimiento de carcasa, aunque en este caso la diferencia fue significativa $(p<0,05)$.

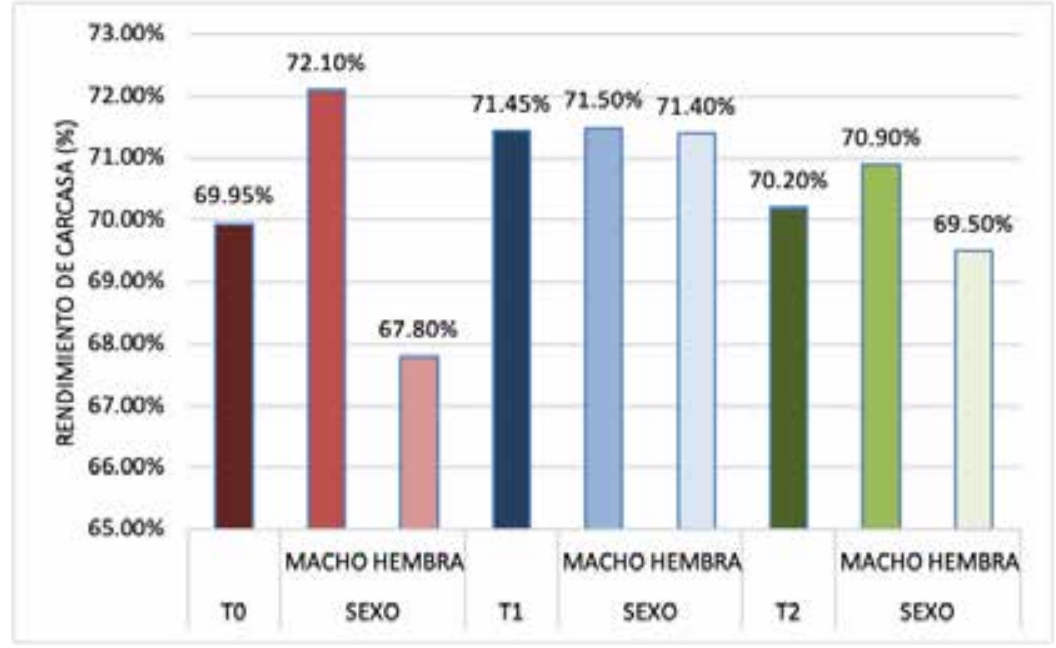

Figura 3. Porcentaje del rendimiento de carcasa por tratamientos y por sexo

\section{Porcentaje de mortalidad}

El mayor porcentaje de mortalidad en este experimento fue en el T2 que tuvo $16,7 \%$ de mortalidad, seguido por el TO con 8,3 \% y finalmente el T1 con $0 \%$. No se calcularon diferencias estadísticas significativas entre los tratamientos $(p>0,05)$. No se encontraron reportes en donde se indique el porcentaje de mortalidad en cuyes al utilizar un promotor natural de crecimiento. 


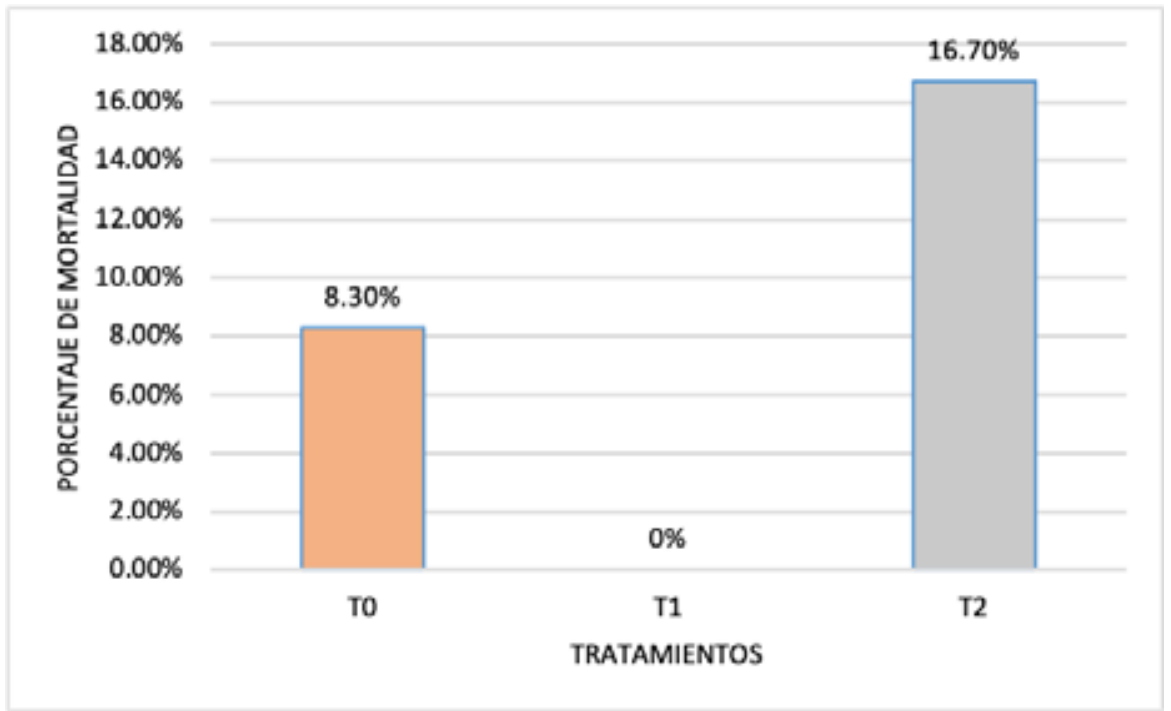

Figura 4. Porcentaje de mortalidad por tratamientos

\section{CONCLUSIONES}

La adición de promotor natural de crecimiento (PNC) en un nivel de $50 \mathrm{~g} / \mathrm{kg}$ de alimento mejoró el incremento de peso (841.5 g), la conversión alimenticia $(3,1)$, el rendimiento de carcasa $(72.5$ $\%) ;$ así como, se presentó un $0 \%$ de mortalidad en cuyes en la etapa de crecimiento - acabado.

Es importante continuar investigando en la búsqueda de alternativas de sustitutos de antibióticos en cuyes por la repercusión negativa que estos representan para la salud del ser humano, así como para los animales.

\section{REFERENCIAS BIBLIOGRÁFICAS}

Aliaga, L.; Moncayo, R.; Elizabeth, E.; Caicedo, A. (2009). Producción de cuyes. Fondo editorial UCSS. Lima - Perú.

Andía Ayme, V., \& Ángeles Lazo, A. M. (2021). Efecto de alimento suplementado con una mezcla probiótica sobre los parámetros productivos de Cavia porcellus, cuy. Revista Tayacaja, 4(2), 13-21. https://doi. org/10.46908/revistatayacaja.v4i2.167

Cano, J. (2012). Efecto de la suplementación de prebiótico liquido sobre los parámetros productivos en cuyes (Cavia porcellus) durante la fase de crecimiento y engorde. Tesis de pregrado. Universidad Nacional
Mayor de San Marcos.

Cano, J., Carcelén, F., Ara, M., Quevedo, W. Alvarado, A. y Jiménez, R. (2015). Efecto de la suplementación con una mezcla probiótica sobre el comportamiento productivo de cuyes (Cavia porcellus) durante la fase de crecimiento y acabado. Disponible en: http://www.scielo.org.pe/ pdf/rivep/v27n1/a07v27n1.pdf

Castro (2002). Formulación de dietas y balanceadas en base a granos de desecho de maíz, trigo y cebada para cuyes. Tesis de la Universidad Técnica del Norte. Ibarra, Ecuador.

Coba, L. (2012). Respuesta de la ganancia de peso con la adición de Promotores de crecimiento en cuyes. México. Disponible en https://prezi.com/edbkd2_ibjOg/respuestade-la-ganancia-de-peso-con-la-adicion-depromotores-de-crecimiento-en-cuyes/

Guevara, J. y Carcelén F. (2014). Efecto de la suplementación de probióticos sobre los parámetros productivos de cuyes. Revista Peruana de Ingeniería Química. Vol. 17, (2). 69-74.Disponible en: http:// revistasinvestigacion.unmsm.edu.pe/index. php/quim/article/download/11332/10162 
Morales, A. (2009). Evaluación de dos niveles de energía en el comportamiento productivo de cuyes de la raza Perú. [Tesis de grado]. Universidad Nacional Mayor de San Marcos. Facultad de Medicina Veterinaria. Lima - Perú.

Morales, M. (2017). Patógenos bacterianos y parasitarios más frecuentes en cuyes de crianza familiar comercial en tres distritos de la Provincia de Bolognesi, Departamento de Ancash en época de seca. [Tesis de grado]. Universidad Nacional Mayor de San Marcos. Disponible en: https://cybertesis. unmsm.edu.pe/handle/20.500.12672/6875

Pozo, V. H.; Tepú, A.H. (2012). Evaluar la influencia de la vitamina "C" en cuyes (Cavia porcellus). de engorde en la comunidad de Guananguicho - Cantón San Pedro de Huaca - Carchi. [Tesis de grado]. Ingeniero Agropecuario. Universidad Técnica del Norte. Ecuador. Disponible en: $\quad$ http://repositorio.utn.edu.ec/ handle/123456789/2683

Ordóñez, R. (2003). Plan de introducción de la carne de cuy en Lima Metropolitana: Estudio de mercado y propuesta empresarial. [Tesis de Magister]. Lima: Pontificia Universidad Católica del Perú. Disponible en: https://tesis.pucp.edu.pe/repositorio/ handle/20.500.12404/598

Otero, M. (2013). Estudio de Factibilidad para la Instalación de un Módulo de Cuyes. Estación Universidad Nacional Pedro Ruiz Gallo. Chiclayo.

Phillips I., M. Casewell., T. Cox., B. De Groot., C. Friis., R. Jones y J. Waddell. (2004). Does the use of antibiotics in food animals pose a risk to human health? A critical review of published data. Journal of Antimicrobial Chemotherapy, 53(1), 28-52. DOI: 10.1093/ $\mathrm{jac} / \mathrm{dkg} 483$

Rey, C. (2008). Fortalecimiento de la cadena productiva del cuy en las provincias de Cajabamba, San Marcos, Celendín,
Cajamarca, Hualgayoc y Cutervo. Disponible en: https://es.scribd.com/document// PERFIL-CUYES-EN-OBSERVACION

Torres, C., Carcelén, F., Ara, M., San Martín, F., Jiménez, R., Quevedo, W. y Rodríguez, J. (2013). Efecto de la suplementación de una cepa probiótica sobre los parámetros productivos del cuy (Cavia porcellus). Revista de Investigación Veterinaria del Perú. 24(4): 433-440. Disponible en: http:// www.scielo.org.pe/pdf/rivep.pdf

V. Ravidran, (2010). Aditivos en la alimentación animal presente y futuro. Institute of food, Nutrition and human healt, Massey University, Palmerston North 444. New Zealand. Disponible en: https://www. produccion-animal.com.ar/informacion_ tecnica/invernada_promotores_ crecimiento/44-10CAP_I.pdf 